Women on the River of Life 



\section{Women on the River of Life}

A Fifty-Year Study of Adult Development

\section{Ravenna M. Helson and Valory Mitchell}

甲 
University of California Press

Oakland, California

(C) 2020 by Ravenna M. Helson and Valory Mitchell

Library of Congress Cataloging-in-Publication Data

Names: Helson, Ravenna M., I925- author. | Mitchell,

Valory, author.

Title: Women on the river of life : a fifty-year study of adult development/Ravenna M. Helsonv and Valory Mitchell.

Description: Oakland, California : University of California Press, [2020] I Includes bibliographical references and index.

Identifiers: LCCN 20200I3 I 59 (print) I LCCN 20200 I 3 I 60 (ebook) I ISBN 9780520299 I 53 (hardback) | ISBN 9780520299160 (paperback) I ISBN 978052097 IOI I (epub)

Subjects: LCSH: Women-United States-Social conditions-Longitudinal studies. I Women-United States-Conduct of life-Longitudinal studies. I Women-Psychology.

Classification: LCC HQI4IO .H455 2020 (print) I LCC HQI4IO (ebook) I DDC 305.420973-dc23

LC record available at https://lccn.loc.gov/20200I 3 I 59

LC ebook record available at https://lccn.loc .gov/20200I3I60

Manufactured in the United States of America

$\begin{array}{llllllllll}29 & 28 & 27 & 26 & 25 & 24 & 23 & 22 & 21 & 20\end{array}$

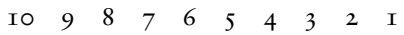

